\section{An example of the application of Mössbauer spectroscopy for determination of concentration of iron in lyophilized brain tissue}

\author{
Patrycja Rzepecka, \\ Przemysław Duda, \\ Joanna Giebułtowicz, \\ Małgorzata Sochacka, \\ Andrzej Friedman, \\ Jolanta Gałązka-Friedman
}

\begin{abstract}
Mössbauer spectroscopy is not routinely used for the determination of the concentration of iron. However, as this method does not need any pre-treatment of samples before measurements, it may be of extreme importance for the assessment of iron in samples, which can then be used for further investigations. Biological samples are a good example, however, as the concentrations of iron are very low in these, it is important to exclude possible artefacts from the background spectrum related to iron present in the counter and cryostat windows. The aim of this study was to compare two methods of determination of the amounts of iron in investigated sample: one, in which the background spectrum was subtracted from the sample spectrum measured, and the other, in which the obtained non-elaborated spectrum was fitted with two doublets - a doublet for the measured sample and a doublet for the background spectrum. Three samples containing known amounts of natural iron $(400,800$ and $1600 \mu \mathrm{g})$ and a sample of lyophilized human brain tissue obtained from globus pallidus were assessed. Both methods led to the creation of a very good calibration curve with a correlation coefficient of 0.99 . Although both methods gave similar results for the concentration of iron in the sample, the subtraction of the background spectrum had a significantly lower error of the final result.
\end{abstract}

Keywords: Mössbauer spectroscopy • background spectrum • iron concentration • brain tissue $\bullet$ calibration curve

P. Rzepecka, P. Duda, J. Gałązka-Friedman ${ }^{\bowtie}$ Faculty of Physics,

Warsaw University of Technology,

75 Koszykowa Str., 00-662 Warsaw, Poland,

E-mail: jgfrie@if.pw.edu.pl

J. Giebułtowicz, M. Sochacka

Faculty of Pharmacy,

Medical University of Warsaw,

Warsaw, Poland

\author{
A. Friedman \\ Department of Neurology, \\ Faculty of Health Science, \\ Medical University of Warsaw, \\ Warsaw, Poland
}

Received: 2 July 2016

Accepted: 29 November 2016

\section{Introduction}

Mössbauer spectroscopy (MS) is not a routine method for the evaluation of the concentration of iron in samples. Due to its great advantage of being a method which does not destroy the sample, it is used when one is dealing with hardly available material, like extraterrestrial samples (e.g., meteorites, lunar soil) or with biological samples, which after the Mössbauer spectroscopy measurements may be assessed by other techniques. The idea of use of MS for the evaluation of concentration of iron was proposed for the first time by Herr and Skerra in 1969 [1]. These authors wanted to use MS as an alternative method for the classification of meteorites. MS was used then by Morris to assess the extraterrestrial samples of lunar soil [2]. In 1994 Bauminger et al. used MS for the first time to determine the concentration of iron in brain samples [3]. With the use of MS, the concentration of iron was determined in more than 100 samples of different human tissues. In 1999, Dubiel [4] applied MS for the assessment of the concentration of iron in one liver and two brain samples. Also, Trautwein [5] used MS to determine the concentration of iron in neuromelanin isolated from brain tissues. In order to determine the concentration of iron based on the analysis of 
the Mössbauer spectra, background effects have to be considered. The main problem related to the use of this method for determining the concentration of iron in biological samples is the very low amount of iron in them. The amount of iron in these samples is comparable to the amount of iron in most counter or cryostat windows. Morris et al. [2] showed that the MS spectrum of iron present in their holder and beryllium windows represents a singlet of $0.54 \mathrm{~mm} / \mathrm{s}$ width and IS $=0.218 \mathrm{~mm} / \mathrm{s}$. The background spectrum from the Mössbauer spectrometer used in Bauminger's lab were presented in [6]. These spectra were measured with statistics of 40 million counts per channel. At room and nitrogen temperature, no signal from background could be detected. At $4.1 \mathrm{~K}$, a weak spectrum from the windows of helium cryostat was observed. In the Jerusalem spectrometer, the counter and nitrogen cryostat had Mylar and not beryllium windows, and thus, it did not contain any iron. A very weak background spectrum at helium temperature was observed, probably because the windows of the helium cryostat were Mylar coated with a thin layer of aluminium, which had enough iron in them to be detected. There is no information about possible impurities from the spectrometer in the paper of Dubiel et al. [4]. In the Trautwein's lab, the published spectra of brain tissues, the sub-spectra from the counter and cryostat window impurities were present [7]. The authors do not comment on the elimination of these artifacts from the obtained spectra.

Before starting to measure, the concentrations of iron in brain samples in the Warsaw lab, we measured the background spectrum with a leucite sample holder filled with boron nitride powder. Two methods were used for the elimination of the impact of these impurities: subtraction of the background spectrum from the spectrum measured (method I) and fitting the non-elaborated spectrum with two doublets - one reflecting the signal from the sample measured and one reflecting the background spectrum (method II). Based on the corrected spectra, a calibration curve showing the area of the spectra vs. the known concentration of iron in the standards used, was displayed. This calibration curve was used for the determination of the amount of iron in the brain sample studied.

\section{Material and method}

The standards for calibration curve were prepared with a homogeneous mixture of iron (III) sulphate and boron nitride powder. Three samples containing known amounts of natural iron - 400, 800, and $1600 \mu \mathrm{g}$ - were assessed. Accurately weighed quantities of the working standards were placed in leucite sample holders. The surface of the holders was typical: $\pi(7 \times 7) \mathrm{mm}^{2}$. A sample of brain tissue was separated from globus pallidus (mass $=1.3 \mathrm{~g}$ ) and lyophilized. Mössbauer measurements were performed at room temperature. A conventional constant acceleration spectrometer with a $3.7 \mathrm{GBq}(100 \mathrm{mCi}){ }^{57} \mathrm{Co}$ source in a $\mathrm{Rh}$ matrix was used. The $14.4 \mathrm{keV}$ gamma rays

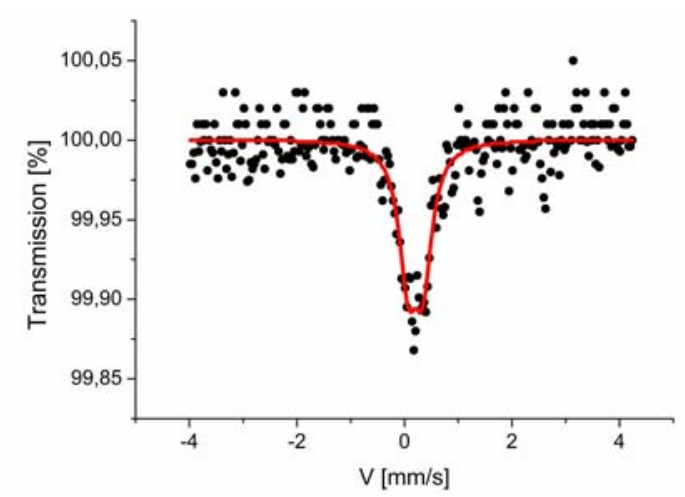

Fig. 1. Mössbauer spectrum of the background.

were detected using a proportional counter (LND INC, model 454106). The velocity scale was calibrated using an iron foil absorber at room temperature. Mössbauer spectra were analysed with the Recoil program.

\section{Results}

In Fig. 1, the background spectrum due to Fe in the counter's Be window, measured at room temperature, is shown.

\section{Method I}

In method I, the background spectrum was subtracted from the sample spectrum measured. For this reason, the coefficient of the normalization was calculated. It was the quotient of the average number of counting in the standard spectrum to the average number of counting in the window spectrum. During the next step, the counter window spectrum was multiplied by the received coefficient. Then it was possible to subtract the window from the standard spectra. Finally, the value of the average number of standard spectra counting was added to the received result. After completing the above presented procedure, an analysis of the Mössbauer spectra was performed using the Recoil program.

In Figs. 2, 3 and 4, Mössbauer spectra of standards containing 400, 800 and $1600 \mu \mathrm{g}$ of iron, before and after subtraction of the background are shown. Spectra of these standards before the subtraction of the background spectrum are asymmetric.

In Fig. 5, the Mössbauer spectrum obtained from the lyophilized sample of globus pallidus (GP) before and after subtraction of the background is shown. Due to the low concentration of iron in brain tissues, the non-subtraction of the background spectrum distorts largely the shape of the spectrum obtained from these tissues.

\section{Method II}

In method II, the obtained non-elaborated spectrum was fitted with two doublets - a doublet for the measured sample and a doublet for the background spectrum. 

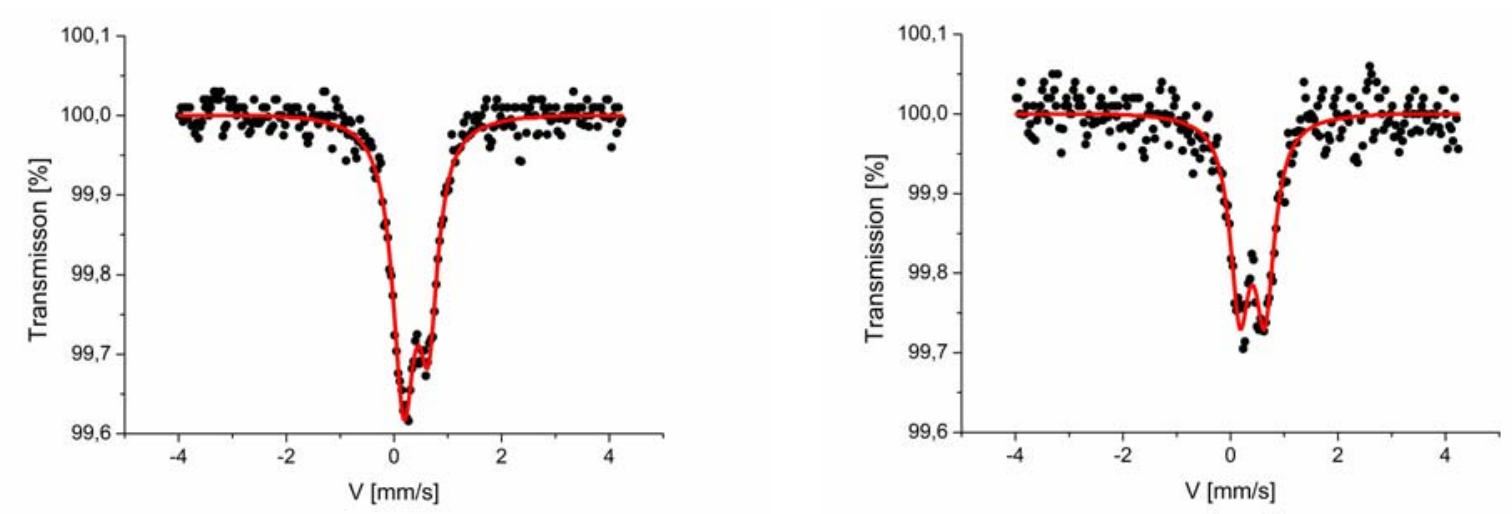

Fig. 2. Mössbauer spectra of standard containing $400 \mu \mathrm{g}$ of natural iron before subtraction of the background spectrum (left) and after it (right).
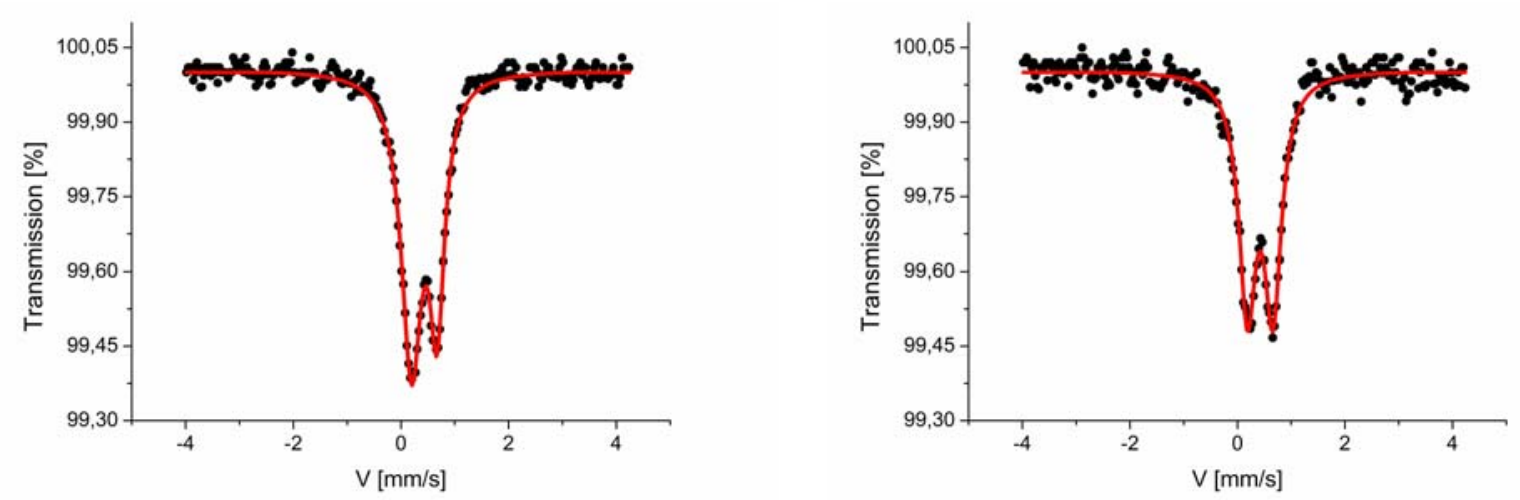

Fig. 3. Mössbauer spectra of a sample containing $800 \mu \mathrm{g}$ of natural iron before subtraction of the background spectrum (left) and after it (right).
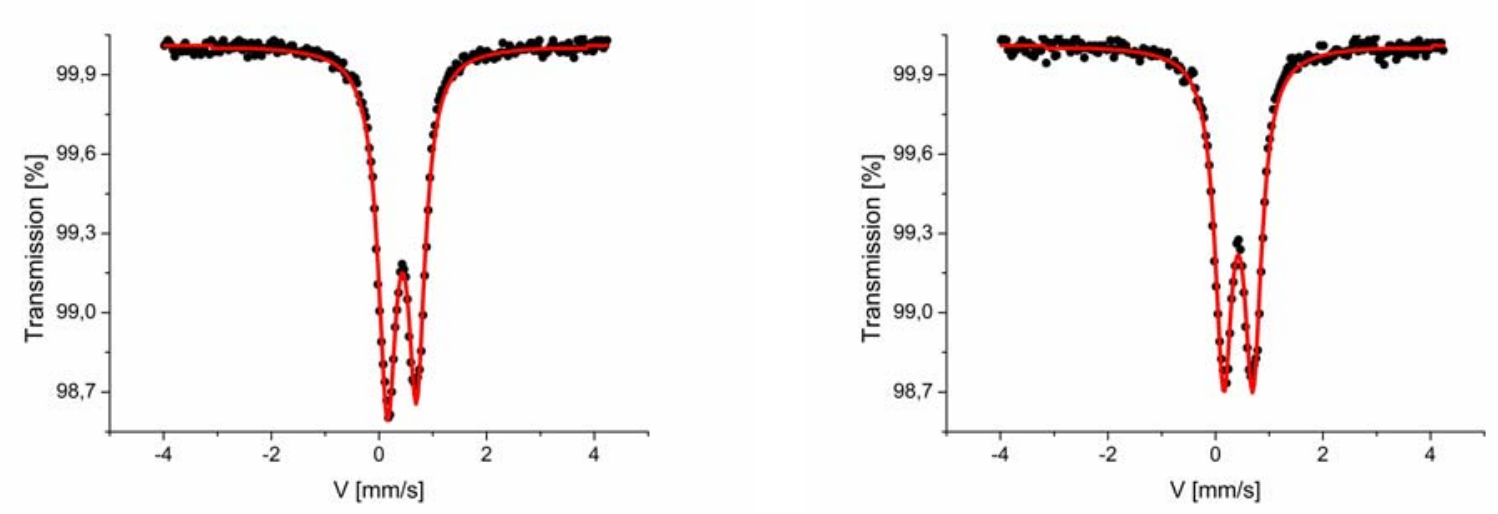

Fig. 4. Mössbauer spectra of a sample containing $1600 \mu \mathrm{g}$ of natural iron before subtraction of the background spectrum (left) and after it (right).
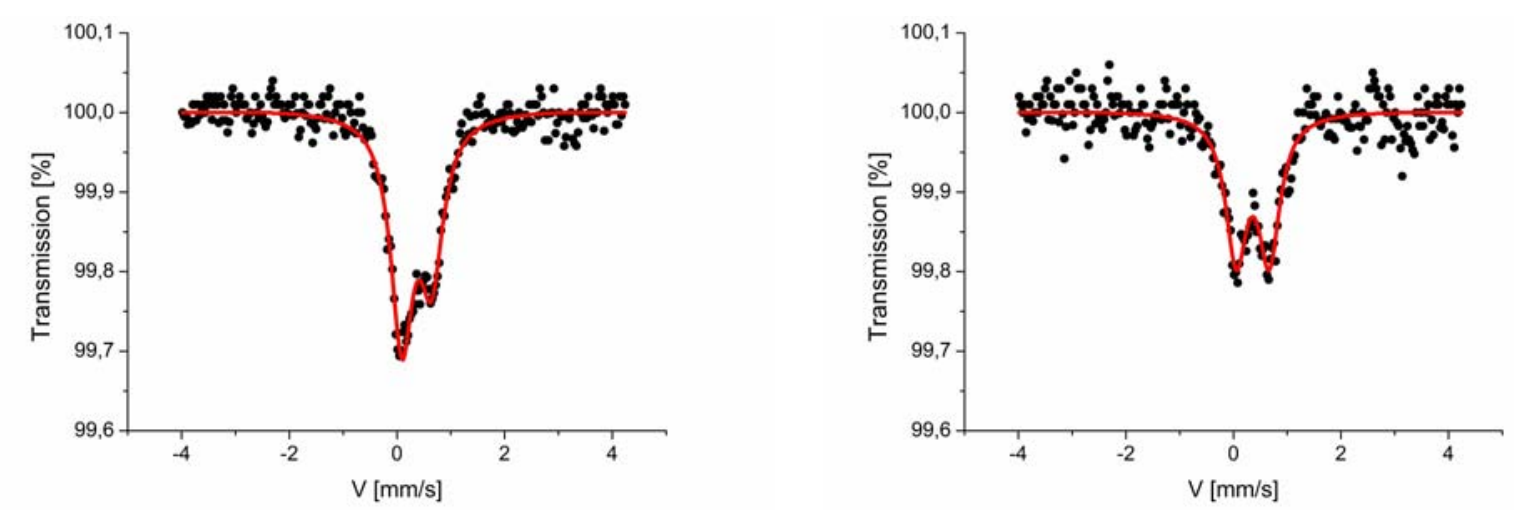

Fig. 5. Mössbauer spectra of a sample of GP with unknown concentration of iron before the subtraction of background spectrum (left) and after it (right). 


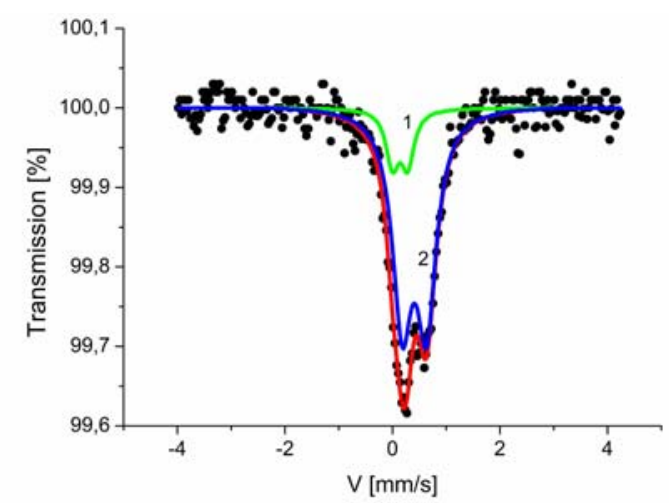

Fig. 6. Mössbauer spectrum of a sample containing $400 \mu \mathrm{g}$ of natural iron fitted with two doublets (background spectrum (1) and a doublet of iron salt (2)).

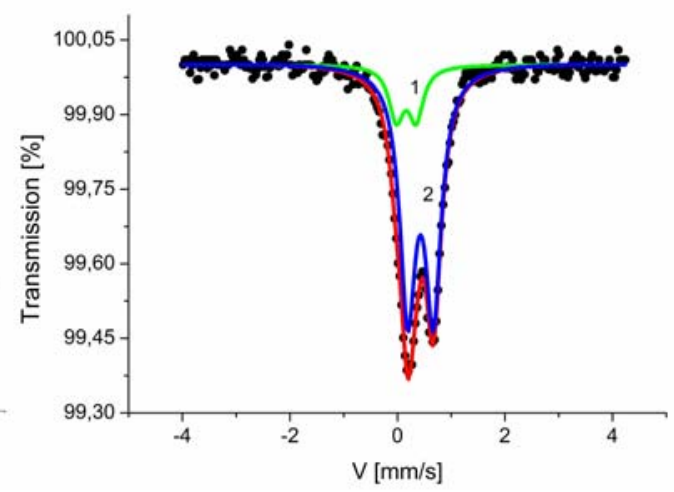

Fig. 7. Mössbauer spectrum of a sample containing $800 \mu \mathrm{g}$ of natural iron fitted with two doublets (background spectrum (1) and a doublet of iron salt (2)).

In Figs. 6, 7, 8 and 9, Mössbauer spectra of standards and the GP sample fitted by two doublets are shown - one doublet with parameters of the background spectrum and the other displaying the obtained spectrum of the samples.

Table 1 summarizes the Mössbauer parameters of the standards and GP obtained after the subtraction of background using method I.

In Figs. 10 and 11, the calibration curve obtained with the use of method I and II is shown. The red spot represents the values obtained for GP.

Table 1. Mössbauer spectra parameters obtained with method I for standards containing 400, 800 and $1600 \mu \mathrm{g}$ of natural iron and for GP. IS - isomer shift, QS - quadrupole splitting, $W$ - HWHM (half width at half maximum)

\begin{tabular}{ccccc}
\hline Sample & $\begin{array}{c}\text { IS } \\
{[\mathrm{mm} / \mathrm{s}]}\end{array}$ & $\begin{array}{c}\text { QS } \\
{[\mathrm{mm} / \mathrm{s}]}\end{array}$ & $\begin{array}{c}W \\
{[\mathrm{~mm} / \mathrm{s}]}\end{array}$ & $\begin{array}{c}\text { Area } \\
{[\text { counts mm/s] }}\end{array}$ \\
\hline GP & $0.36 \pm 0.03$ & $0.62 \pm 0.04$ & $0.24 \pm 0.04$ & $(472 \pm 36) \times 10^{2}$ \\
400 & $0.41 \pm 0.02$ & $0.46 \pm 0.03$ & $0.22 \pm 0.03$ & $(661 \pm 36) \times 10^{2}$ \\
800 & $0.42 \pm 0.02$ & $0.48 \pm 0.02$ & $0.19 \pm 0.02$ & $(1354 \pm 36) \times 10^{2}$ \\
1600 & $0.43 \pm 0.02$ & $0.55 \pm 0.02$ & $0.19 \pm 0.02$ & $(2861 \pm 33) \times 10^{2}$ \\
\hline
\end{tabular}

Table 2. Mössbauer spectra parameters obtained with method II for standards containing 400, $800 \mu \mathrm{g}$ natural iron and $1600 \mu \mathrm{g}$ and for GP. IS - isomer shift, QS - quadrupole splitting, $W$ - HWHM (half width at half maximum)

\begin{tabular}{ccccc}
\hline Sample & $\begin{array}{c}\text { IS } \\
{[\mathrm{mm} / \mathrm{s}]}\end{array}$ & $\begin{array}{c}\text { QS } \\
{[\mathrm{mm} / \mathrm{s}]}\end{array}$ & $\begin{array}{c}W \\
{[\mathrm{~mm} / \mathrm{s}]}\end{array}$ & $\begin{array}{c}\text { Area } \\
{[\text { counts mm/s] }}\end{array}$ \\
\hline GP & $0.39 \pm 0.04$ & $0.59 \pm 0.05$ & $0.22 \pm 0.05$ & $(460 \pm 146) \times 10^{2}$ \\
400 & $0.41 \pm 0.03$ & $0.45 \pm 0.04$ & $0.22 \pm 0.04$ & $(739 \pm 109) \times 10^{2}$ \\
800 & $0.43 \pm 0.02$ & $0.48 \pm 0.02$ & $0.18 \pm 0.02$ & $(1312 \pm 130) \times 10^{2}$ \\
1600 & $0.43 \pm 0.02$ & $0.54 \pm 0.02$ & $0.19 \pm 0.02$ & $(2908 \pm 55) \times 10^{2}$ \\
\hline
\end{tabular}




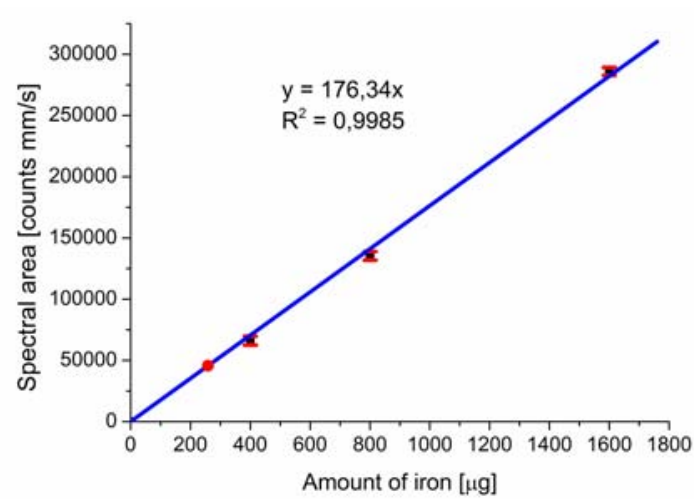

Fig. 10. Area under Mössbauer spectra of standards vs. the amount of iron in investigated samples (parameters obtained with the use of method I).

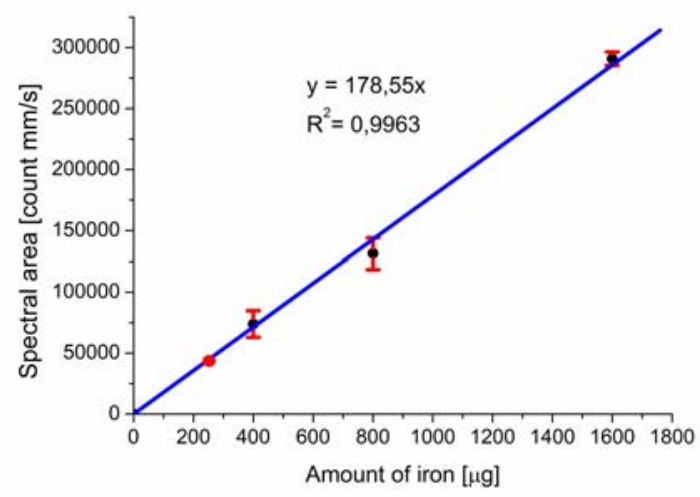

Fig. 11. Area under Mössbauer spectra of standards vs. the amount of iron in investigated samples (parameters obtained with the use of method II).

\section{Discussion}

Both methods used for the elimination of signal from impurities - the subtraction of background spectrum and the fitting of obtained spectrum with two doublets (one representing the investigated sample, and other representing the background spectrum) - seem to be equivalent as they lead to the correct Mössbauer parameters of the assessed samples (iron salts used for preparation of standards and ferritin in the globus pallidus).

Both methods led to the creation of a very good calibration curve with correlation coefficient equal to 0.99 .

The subtraction of background spectrum method (method I) gives a significantly lower error for the final result of concentration of iron in the sample than fitting with the two doublets (method II) - 8\% vs. $32 \%$.

The Mössbauer parameters obtained from the brain tissue are identical (within the error limits) to those from laboratories of Bauminger [3], Trautwein [7] and Miglierini [8]. They are, however, different than those from Dubiel et al. [4]. In this paper, the background was probably neglected. However, even in iron rich samples with a lot of sub-spectra (e.g., in meteorite samples), the background might distort the analysis.

\section{Conclusions}

While planning systematic studies of biological samples, the use of an equipment which does not give an additional background signal has to be preferred; however, unfortunately, at present, no proportional counters with windows are available that do not contain iron.

This method of determining the concentration of iron in biological samples will need a correction related to the value of Debye-Waller coefficient. In future, we plan to prepare standards made from ferritin and measure them as a function of temperature.

Acknowledgment. We would like to thank Professor Erika R. Bauminger for a very fruitful discussion of this manuscript.

\section{References}

1. Herr, W., \& Skerra, B. (1969). Mössbauer spectroscopy applied to the classification of stone meteorites. In: P. M. Millman (Ed.), Meteorite research (Vol. 12, pp. 106-122). Astrophysics and Space Series. Science Library. Dordrecht: D. Reidel Pub. Co.

2. Morris, R. V., Klingelhöfer, G., Korotev, L. R., \& Shelfer, T. D. (1998). Mössbauer mineralogy on the Moon: The lunar regolith. Hyperfine Interact., 117, 405-432.

3. Bauminger, E. R., Barcikowska, M., Friedman, A., Hechel, D., \& Nowik, I. (1994). Does iron play a role in Parkinson's disease? Hyperfine Interact., 91, 853-856.

4. Dubiel, S. M., Zabłotna-Rypień, B., Mackey, J. B., \& Williams, J. M. (1999). Magnetic properties of human liver and brain ferritin. Eur. Biophys. J., 28, 263-267.

5. Gerlach, M., Trautwein, A. X., Zecca, L., Youdim, M. B. H., \& Riederer, P. (1995). Mössbauer spectroscopic studies of purified human neuromelanin isolated from the substantia nigra. J. Neurochem., 65, 923-926.

6. Galazka-Friedman, J., Bauminger, E. R., Koziorowski, D., \& Friedman, A. (2004). Mössbauer spectroscopy and ELISA studies reveal differences between Parkinson's disease and control substantia nigra. Biochim. Biophys. Acta, 1688, 130-136.

7. Zecca, L., Gallorini, M., Schunemann, V., Trautwein, A. X., Gerlach, M., Riederer, P., Vezzoni, P., \& Tampellini, D. (2001). Iron, neuromelanin and ferritin content in the substantia nigra of normal subjects at different ages: consequences for iron storage and neurodegenerative processes. J. Neurochem., 76(6), 1766-1773.

8. Miglierini, M., Rocca, R., Kopani, M., \& Lancok, A. (2014). Mössbauer and SQUID characterization of iron in human tissue: case of globus pallidus. Acta Phys. Pol. A, 126, 240-241. 